\title{
MAGNETOELECTRIC SENSORS OF MAGNETIC FIELD BASED ON LAMINATED STRUCTURES WITH COMPOSITE MAGNETOSTRICTIVE LAYERS
}

\author{
A. P. Nosov $^{1}$, I. V. Gribov ${ }^{1}$, N. A. Moskvina ${ }^{1}$, A. V. Druzhinin ${ }^{1}$, V. I. Osotov ${ }^{1}$, \\ V. A. Bespalov², B. A. Loginov ${ }^{2}$ \\ ${ }^{1}$ M.N. Miheev Institute of Metal Physics of Ural Branch of Russian Academy of Sciences, \\ 18, S. Kovalevskoy st., Ekaterinburg, Russian Federation \\ ${ }^{2}$ National Research University of Electronic Technology MIET, Moscow, Zelenograd, Russian Federation \\ *Corresponding author. E-mail: nossov@imp.uran.ru; address for correspondence: ul. S. Kovalevskoy 18, \\ 620990, Ekaterinburg, Russia. Tel.: +7 (343) 37479 22; fax: +7 (343) 3745244.
}

The magnetoelectric effect (ME) in three-layered laminated structures of the "composite magnetostrictive ferromagnetic material / ferroelectric / composite magnetostrictive ferromagnetic material" type is investigated. The composite magnetostrictive ferromagnetic material consists of "Metglas"-type amorphous ribbons on which magnetostrictive $\mathrm{Fe}_{0.72} \mathrm{Ga}_{0.28}$ or $\mathrm{Fe}_{0.62} \mathrm{Co}_{0.19} \mathrm{Ga}_{0.19}$ thin films are deposited by pulsed laser deposition. The ME is investigated for $d c$ and $a c$ magnetic fields in the frequency range from $20 \mathrm{~Hz}$ to $10 \mathrm{kHz}$. It is shown experimentally that the deposition of the $\mathrm{Fe}_{0.72} \mathrm{Ga}_{0.28}$ films increases the maximum value of the ME in laminated structures in the whole frequency range investigated, while the deposition of the $\mathrm{Fe}_{0.62} \mathrm{Co}_{0.19} \mathrm{Ga}_{0.19}$ films decreases this value. The laminated structures with $\mathrm{Fe}_{0.72} \mathrm{Ga}_{0.28}$ films operating under excitation with the frequency of $110 \mathrm{~Hz}$ are the most appropriate for applications in magnetic nondestructive testing.

Keywords: nondestructive testing, magnetic field sensor, magnetoelectric effect, amorphous alloy, thin films, galfenol.

DOI: $10.17804 / 2410-9908.2016 .5 .030-038$

\section{References}

1. Romashev L., Rinkevich A., Yuvchenko A., Burkhanov A. Magnetic field sensors based in Fe/Cr superlattices. Sensors and Actuators A: Physical, 2001, vol. 91, iss. 1-2, pp. 30-33. DOI: 10.1016/S0924-4247(01)00482-4.

2. Naumova L.I., Milyaev M.A., Chernyshova T.A., Proglyado V.V., Kamenskii I.Yu., Ustinov V.V. Hysteresis-free spin valves with a noncollinear configuration of magnetic anisotropy. Physics of the Solid State, vol. 56, iss. 6, pp. 1125-1130. DOI: 10.1134/S1063783414060274.

3. Lawes G., Srinivasan G. Introduction to magnetoelectric coupling and multiferroic films. J. Phys. D: Appl. Phys, 2011, vol. 44, pp. 243001 (22). DOI:10.1088/0022-3727/44/24/243001.

4. Petrov V.M., Srinivasan G. Enhancement of magnetoelectric coupling in functionally graded ferroelectric and ferromagnetic bilayers. Phys. Rev. B, 2008, vol. 78, pp. 184421 (8). DOI: 10.1103/PhysRevB.78.184421.

5. Mandal S.K., Sreenivasulu G., Petrov V.M., Srinivasan G. Flexural deformation in a compositionally stepped ferrite and magnetoelectric effects in a composite with piezoelectrics. Appl. Phys. Lett., 2010, vol. 96, pp. 192502 (3). DOI: 10.1063/1.3428774.

6. Magnetic Alloy 2605SA1 (iron-based). Tech. Bulletin, ref: 2605SA106192009. Metglas Inc., Conway, SC, 2009.

7. Passamani E.C., Larica C., Moscon P.S., Mendoza Zelis P., Sanchez F.H. Out-of-plane anisotropy and low field induced magnetic domain reorientation in Al/Metglas-2605S2/Al trilayer sensors. J. Appl. Phys., 2010, vol. 110, pp. 043906 (7). DOI: 10.1063/1.3622339.

8. Gammamet ${ }^{\circledR}$. Research and Production Enterprise. (In Russian). Available at: http://www.gammamet.ru/ru/gm440a.htm 
9. Atulasimha Jayasimha, Flatau Alison B. Topical Review. A review of magnetostrictive iron-gallium alloys. Smart Materials and Structures, 2011, vol. 20, no. 4, pp. 043001 (15). DOI:10.1088/09641726/20/4/043001.

10. Jen S.U., Tsai T.L., Kuo P.C., Chi W.L., Cheng W. C. Magnetostrictive and structural properties of FeCoGa films. J. Appl. Phys., 2010, vol. 107, iss. 1, pp. 013914 (4). DOI: 10.1063/1.3284962.

11. OptoSystems. The leading manufacturer of lasers for medicine, science and technology in Russia. (In Russia). Available at: http://www.optosystems.ru/eng/index.php.

12. Sreenivasulu G., Petrov V.M., Fetisov L.Y., Fetisov Y.K., Srinivasan G. Magnetoelectric interactions in layered composites of piezoelectric quartz and magnetostrictive alloys. Physical Review B, 2012, vol. 86, pp. 214405 (7). DOI: 10.1103/PhysRevB.86.214405.

13. Van der Burgt C.M. Dynamical parameters in the magneto-strictive excitation of extensional and torsional vibrations in ferrites. Philips Res. Rep., 1953, vol. 8, pp. 91-132.

14. Morley N.A., Javed A., Gibbs M.R. Effect of a forming field on the magnetic and structural properties of thin Fe-Ga films. J. Appl. Phys., 2009, vol. 105, pp. 07A912 (4). DOI: 10.1063/1.3059612.

15. Callen Herbert B., Goldberg N. Magnetostriction of Polycrystalline Aggregates. J. Appl. Phys., 1965, vol. 36, pp. 976-977. DOI: 10.1063/1.1714285.

16. Wuttig Manfred, Dai Liyang, Cullen J. Elasticity and magnetoelasticity of Fe-Ga solid solutions. App. Phys. Lett., 2002, vol. 80, pp. 1135 (3). DOI: 10.1063/1.1450045. 
Подана в журнал: 11.10 .2016

УДК 537.633.9

DOI: $10.17804 / 2410-9908.2016 .5 .030-038$

\title{
МАГНИТОЭЛЕКТРИЧЕСКИЕ ДАТЧИКИ МАГНИТНОГО ПОЛЯ НА ОСНОВЕ ЛАМИНАТНЫХ СТРУКТУР С КОМПОЗИТНЫМИ МАГНИТОСТРИКЦИОННЫМИ СЛОЯМИ
}

\author{
А. П. Носов ${ }^{1}$, И. В. Грибов ${ }^{1}$, Н. А. Москвина ${ }^{1}$, А. В. Дружинин ${ }^{1}$, В. И. Осотов ${ }^{1}$, \\ В. А. Беспалов ${ }^{2}$, Б. А. Логинов ${ }^{2}$ \\ ${ }^{l}$ Федеральное государственное бюджетное учреждение науки Институт физики металлов \\ имени М.Н. Михеева Уральского отделения Российской академии наук, 620990, ул. С. Ковалевской 18, \\ Екатеринбург, Россия \\ ${ }^{2}$ Национальный исследовательский университет «МИЭТ», Москва, Зеленоград, Россия \\ *Ответственный автор. Электронная почта: nossov@imp.uran.ru; адрес для переписки: ул. С. Ковалевской, 18, \\ 620990,Екатеринбург, Россия; Телефон: +7 (343) 374-79-22; факс: +7 (343) 374-52-44.
}

Исследован магнитоэлектрический эффект (МЭ) в трехслойных ламинатных структуpax типа композитный магнитострикционный ферромагнетик /сегнетоэлектрик/ композитный магнитострикционный ферромагнетик. Композитный магнитострикционный ферромагнетик состоял из ленты аморфного сплава типа «Метглас» на поверхность которой импульсным лазерным осаждением были нанесены тонкие магнитострикционные пленки составов $\mathrm{Fe}_{0,72} \mathrm{Ga}_{0,28}$ или $\mathrm{Fe}_{0,62} \mathrm{Co}_{0,19} \mathrm{Ga}_{0,19}$. Исследованы зависимости магнитоэлектрического эффекта в ламинатных структурах от статических и переменных магнитных полей в диапазоне частот от 20 Гц до 10 кГц. Экспериментально показано, что нанесение магнитострикционных пленок состава $\mathrm{Fe}_{0,72} \mathrm{Ga}_{0,28}$ приводит к увеличению, а пленок состава $\mathrm{Fe}_{0,62} \mathrm{Co}_{0,19} \mathrm{Ga}_{0,19}$ - к уменьшению максимальной величины магнитоэлектрического эффекта в ламинатных структурах во всем исследованном интервале частот. Для решения задач магнитного неразрушающего контроля наилучшую чувствительность могут обеспечить ламинатные структуры с магнитострикционными пленками состава $\mathrm{Fe}_{0,72} \mathrm{Ga}_{0,28}$ в условиях возбуждения переменным полем с частотой 110 Гц.

Ключевые слова: неразрушающий контроль, датчик магнитного поля, магнитоэлектрический эффект, аморфный сплав, тонкие пленки, галфенол.

\section{1. Введение}

Магнитные методы играют важную роль в решении актуальных проблем неразрушающего контроля, прогнозировании ресурса и испытаний современных конструкционных и функциональных материалов. Ключевые параметры приборов магнитного неразрушающего контроля определяются характеристиками используемых датчиков, действие которых основано на различных физических эффектах. В последние годы при регистрации полей рассеяния дефектов активно используют датчики, принцип действия которых основан на эффекте гигантского магнитосопротивления [1], а также спиновые клапаны [2]. Между тем, для этих целей также может быть использован магнитоэлектрический эффект (МЭ) в композитных планарных структурах с механически связанными слоями из магнитострикционного ферромагнитного и сегнетоэлектрического материалов [3]. Если такую структуру поместить в статическое магнитное поле $d H_{a c}$, то в присутствии переменного магнитного поля $d H_{a c}$ будет наблюдаться прямой МЭ. При воздействии магнитных полей за счет магнитострикции в ферромагнитном слое возникнет механическая деформация, которая приведет к генерации зарядов и возникновению электрического поля $d E$ на поверхности структуры вследствие пьезоэлектрических свойств сегнетоэлектрического слоя. Величина МЭ характеризуется магни- 
тоэлектрическим коэффициентом по напряжению (МЭКН), который определяют как $\alpha=d E / H_{a c}=d V /\left(t d H_{a c}\right)$, где $d V-$ величина возникающего электрического напряжения, $t-$ толщина сегнетоэлектрического слоя. Значение $\alpha$ определяется физическими характеристиками как ферромагнитного слоя, в частности пьезомагнитным коэффициентом $q=d \lambda / d H$, где $\lambda$ - коэффициент магнитострикции; $H$ - статическое магнитное поле, так и сегнетоэлектрического слоя, в частности пьезомодулем $d$ и относительной диэлектрической проницаемостью $\varepsilon$. При использовании МЭ-структур в качестве датчиков магнитных полей в магнитном неразрушающем контроле важна большая величина МЭКН, которая может быть достигнуто оптимизацией характеристик магнитострикционной подсистемы за счет величины $q$. Теоретически это было показано в работе [4]. Для композитных объемных структур с ферромагнитными слоями на основе тонких лент эти результаты получили экспериментально подтверждение в работе [5].

В качестве магнитострикционных слоев композитных МЭ-структур очень часто используются аморфные магнитострикционные ленты из сплавов класса «Метглас» (Metglas) [6]. Это класс материалов характеризуется большим значением q, малым коэрцитивным полем, большой начальной магнитной проницаемостью, положительным значением $\lambda$. Принципиально возможно улучшение магнитострикционных свойств аморфных лент этого класса нанесением тонких пленок магнитострикционных материалов, имеющих другой знак коэффициента магнитострикции, т.е. получением композитных структур с градиентом $q$ по толщине. Ранее были исследованы возможности изменения магнитных свойств аморфных лент сплавов типа «Метглас» при нанесении на них тонких пленок немагнитных металлов [7].

В настоящей работе изучен МЭКН в трехслойных ламинатных структурах типа композитный магнитострикционный ферромагнетик /сегнетоэлектрик/ композитный магнитострикционный ферромагнетик на основе ферромагнитных магнитострикционных аморфных лент сплава типа «Метглас» с магнитострикционными пленками $\mathrm{Fe}_{0.72} \mathrm{Ga}_{0.28}$ и $\mathrm{Fe}_{0,62} \mathrm{Co}_{0,19} \mathrm{Ga}_{0,19}$.

\section{2. Материал и методика}

В качестве сегнетоэлектрических слоев использовали пластины сегнетоэлектрической керамики PZT (\#851, APC International, Pennsylvania, USA) с размерами $5 \times 5 \times 0,3$ мм³ $^{3}$ В качестве магнитострикционного сплава класса «Метглас» использовали аморфные ленты состава 440A [8] (компания «Гаммамет», Екатеринбург, Россия) толщиной 34 мкм без термообработок. Статические измерения магнитострикции были проведены с использованием тензодатчика. Измеряли значения коэффициента магнитострикции насыщения при двух ориентациях магнитного поля $H$ : вдоль $\left(\lambda_{11}=+20 \times 10^{-6} / Э\right)$ и поперек $\left(\lambda_{12}=-15 \times 10^{-6} / Э\right)$ направления прокатки. Из этих данных была определена величина пьезомагнитного коэффициента $q=q_{11}+q_{12}=$ $d \lambda_{11} / d H+d \lambda_{12} / d H$. Для лент состава 440 А было получено значение $q=+0.46 \times 10^{-6} / Э$.

Композитную структуру магнитострикционного слоя получали нанесением на поверхность аморфной ленты состава 440A тонких пленок $\mathrm{Fe}_{0,72} \mathrm{Ga}_{0,28}$ или $\mathrm{Fe}_{0,62} \mathrm{Co}_{0,19} \mathrm{Ga}_{0,19}$ методом импульсного лазерного осаждения. Выбор этих составов обусловлен тем, что для объемных материалов системы $\mathrm{Fe}_{1-X} \mathrm{Ga}_{X}$ (галфенол) максимальную величину коэффициента магнитострикции имеют сплавы с $X=0,19$ или 0,28 [9]. Магнитострикция в сплавах $\mathrm{Fe}_{1-\mathrm{x}} \mathrm{Gax}$ отрицательна. Легирование галфенола кобальтом позволяет увеличить значение $\lambda$. По данным работы [10] для тонких пленок сплавов Fe-Co-Ga максимальное значение $\lambda$ имел состав $\mathrm{Fe}_{0,62} \mathrm{Co}_{0,19} \mathrm{Ga}_{0,19}$. Пленки толщиной 25 нм наносили с использованием эксимерного лазера $\mathrm{CL}$ 7050 (компания «Оптисистемы», г. Троицк, Московская область, Россия [11]).

Были исследованы трехслойные ламинатные структуры, в которых средним сегнетоэлектрическим слоем являлась пьезоекрамика PZT. В качестве верхнего и нижнего слоев использовали либо аморфные магнитострикционные ленты 440А (далее - образцы 440А) либо аморфные магнитострикционные ленты $440 \mathrm{~A}$ с пленкой $\mathrm{Fe}_{0,72} \mathrm{Ga}_{0,28}$ (далее - образцы 
440A/FeGa), либо аморфные магнитострикционные ленты 440A с нанесенной пленкой $\mathrm{Fe}_{0,62} \mathrm{Co}_{0,19} \mathrm{Ga}_{0,19}$ (далее - образцы $440 \mathrm{~A} / \mathrm{FeCoGa}$ ). Ламинатную структуру формировали склеиванием слоев клеем на основе цианокрилата. Ранее в [12] было показано, что в трехслойных структурах величина МЭКН больше, чем в двухслойных.

Для исследования морфологии поверхности полученных структур использовался сканирующий зондовый микроскоп СММ-2000 (Завод ПРОТОН, г. Зеленоград, Россия). Измерения проводили методом атомно-силовой микроскопии (АСM) в полуконтактном режиме с использованием кантилеверов с типичным радиусом острия менее 10 нм.

МЭ исследовали при приложении к ламинатной структуре статического $H$ и переменного $H_{a c}$ магнитных полей. Напряжение $d V$, возникающее в сегнетоэлектрическом слое, измеряли синхронным детектором. Величину МЭКН вычисляли по формуле $\alpha=d E / H_{a c}=d V /\left(t d H_{a c}\right)$, где $t-$ толщина сегнетоэлектрического слоя. Исследовали изменения $\alpha$ в зависимости от величины статического магнитного поля $H$ и частоты $f$ переменного магнитного поля.

Все измерения были выполнены при комнатной температуре.

\section{3. Результаты и обсуждение}

АСМ исследования поверхности лент 440А до нанесения пленок не выявили явно выраженных элементов рельефа. Среднеквадратичное значение шероховатости $R q$ составляло 0,07 HM.

На рис. 1 представлено АCM изображение поверхности образца 440A/FeGa. После нанесения пленки среднеквадратичное значение шероховатости $R q$ увеличилось до величины 4,80 нм за счет формирования в процессе роста характерных островков (включений) овальной формы.

Типичные характеристики включений: высота - порядка 20-25 нм, ширина у основания - порядка 0,2 мкм, расстояние между включениями - порядка 0,4-0,5 мкм. Формирование островковой структуры связано с механизмом роста пленки типа Странски-Крастанов (Stranski-Krastanov). Поскольку температура роста была достаточно низкой $\left(200{ }^{\circ} \mathrm{C}\right)$ то энергия поверхностной диффузии атомов пленки оказалась недостаточной для послойного роста по механизму Франка-ван дер Мерве (Frank-van der Merve).

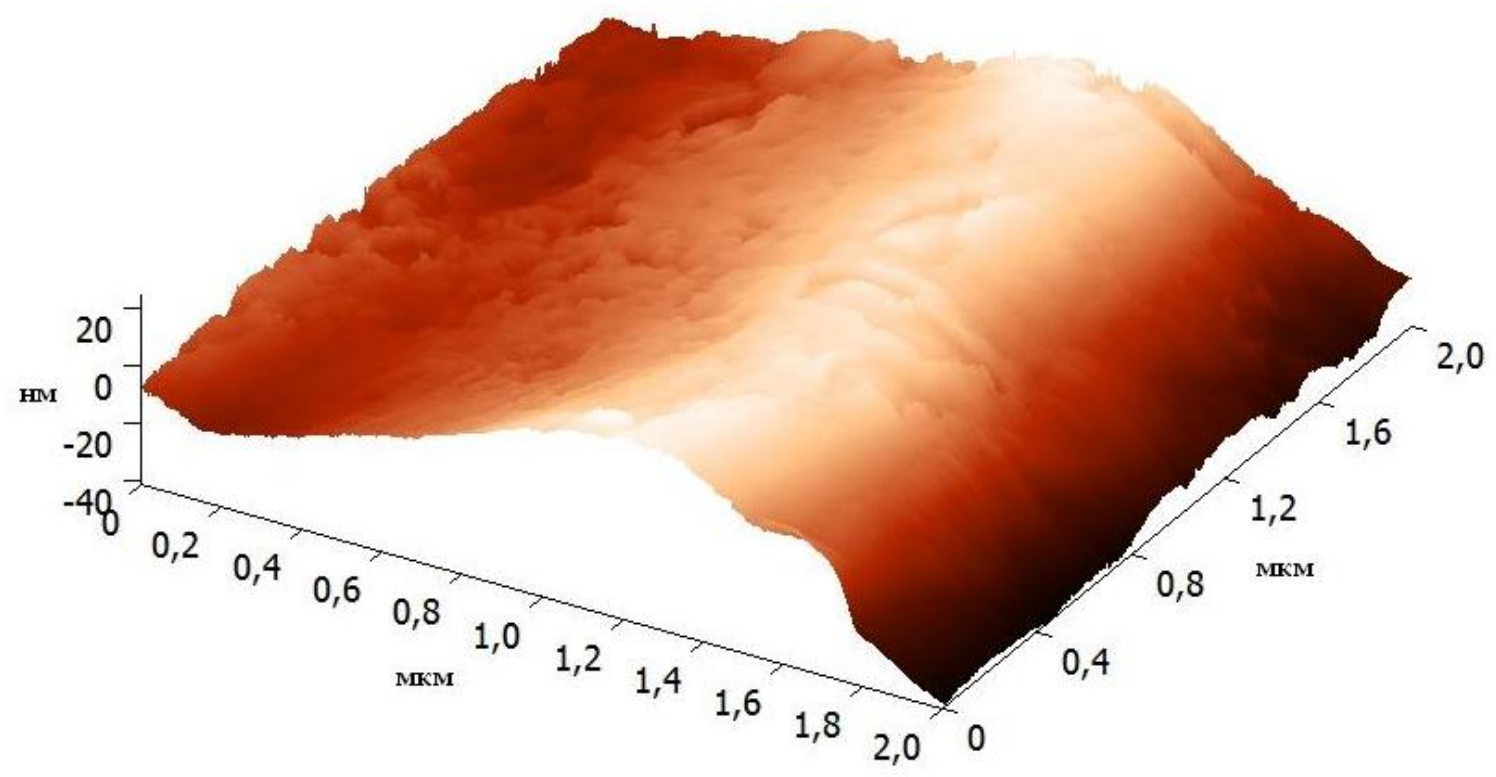

Рис. 1. АСМ изображение поверхности образца 440A/FeGa. 
На рис. 2 приведены результаты, полученные для структуры с аморфной лентой 440A на частотах 110, 500 Гц и 10 кГц. На всех зависимостях $\alpha(H)$ имеется максимум при $H \approx 58$ Э, соответствующий максимуму полевой зависимости коэффициента $q$. Положение максимума практически не зависит от частоты, в то время величина $\alpha_{\text {max }}$ при $H \approx 58$ Э сильно изменяется с частотой. С ростом $H$ значения $\alpha$ постепенно уменьшаются и становятся очень малыми при $H>200 Э$. Ход зависимости $\alpha(H)$ качественно соответствует виду зависимости $q(H)$. Для структур с пленками $\mathrm{Fe}_{0,72} \mathrm{Ga}_{0,28}$ или $\mathrm{Fe}_{0,62} \mathrm{Co}_{0,19} \mathrm{Ga}_{0,19}$ были получены аналогичные по форме зависимости $\alpha(H)$. Нанесение пленок не изменяло положение максимумов зависимостей $\alpha_{\max }(f)$ при $H \approx 58$ Э, однако влияло на величину максимального значения $\alpha$ во всем исследованном частотном диапазоне. Для решения задач магнитного неразрушающего контроля существенно, что величина МЭКН линейно зависит от $H$ в области полей 20-50 Э. Кроме того, зависимости $\alpha(H)$ нечетны по полю, что позволяет по знаку МКЭН судить об ориентации датчика относительно $H$.

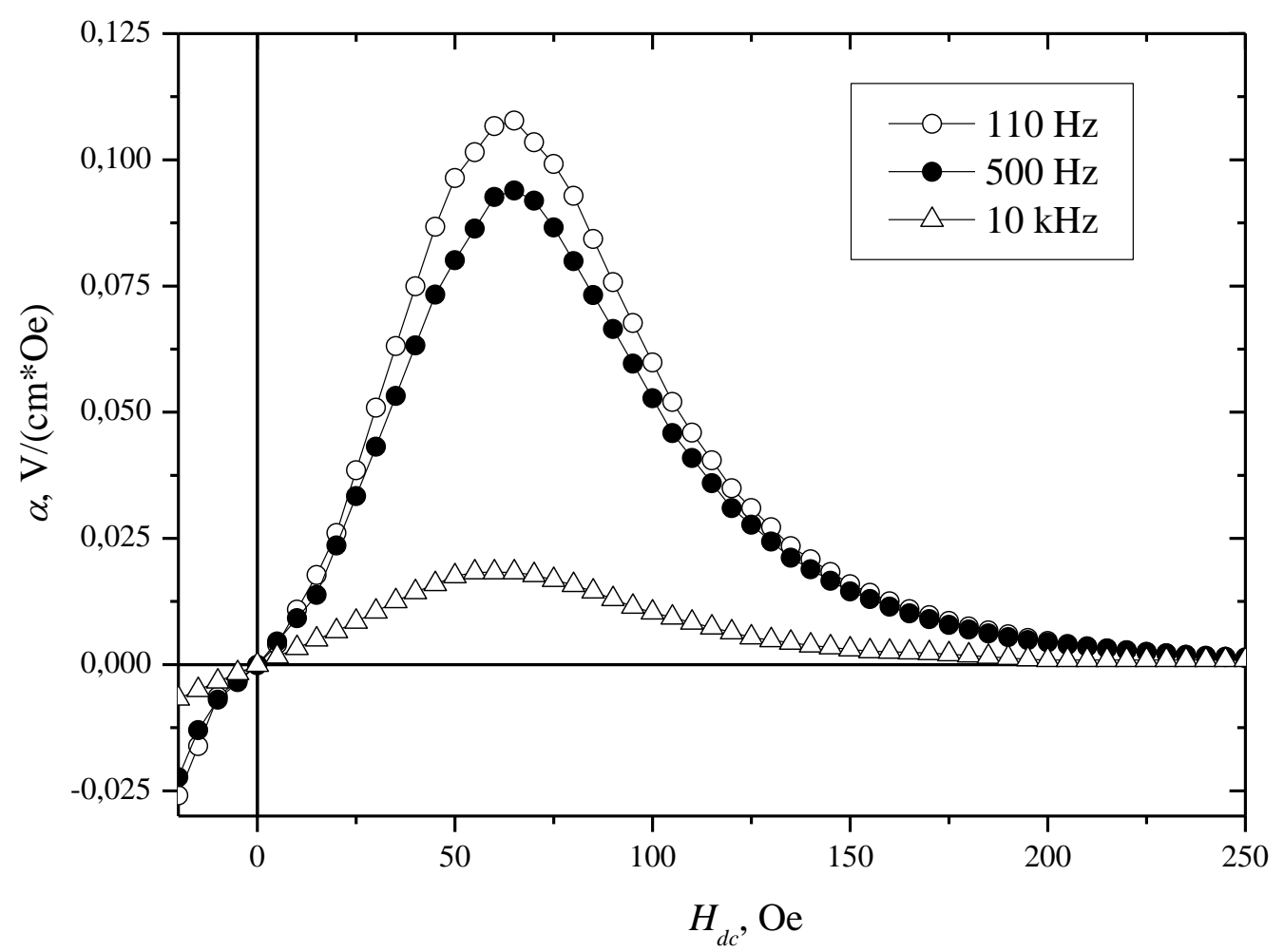

Рис. 2. Зависимости МЭКН от статического магнитного поля для значений частот переменного магнитного поля 110 Гц, 500 Гц и 10 кГц.

Данные для структуры с аморфной лентой 440A

Частотные зависимости МЭКН в поле $H=58$ Э для исследованных образцов приведены на рис. 3 , из которого видно, что нанесение пленки состава $\mathrm{Fe}_{0,72} \mathrm{Ga}_{0,28}$ приводит к небольшому росту $\alpha_{\max }(f)$ во всем частотном диапазоне, а нанесение пленки состава $\mathrm{Fe}_{0,62} \mathrm{Co}_{0,19} \mathrm{Ga}_{0,19}$ приводит к существенному уменьшению значений $\alpha_{\text {max }}(f)$. 


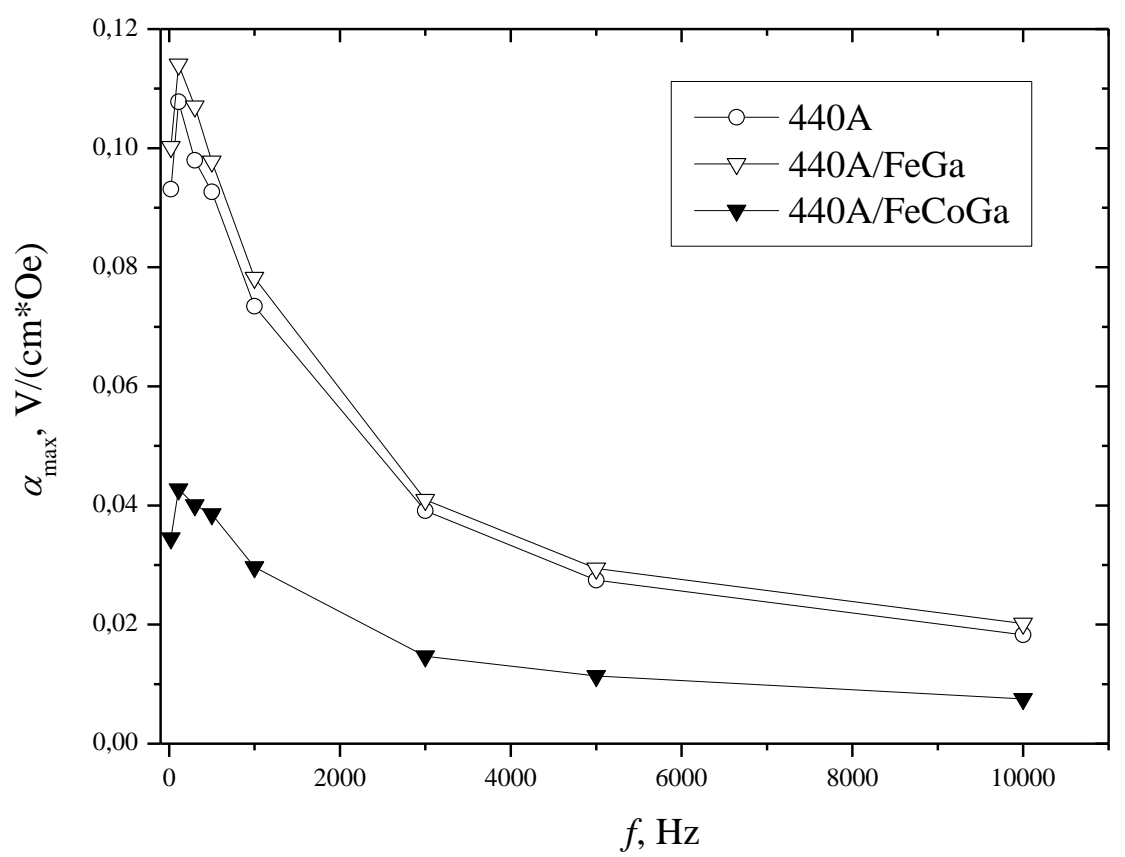

Рис. 3. Сравнение частотных зависимостей максимальной величины МЭКН для структур с магнитострикционными слоями только с аморфной лентой (440A) и аморфными лентами с пленками $\mathrm{Fe}_{0,72} \mathrm{Ga}_{0,28}(440 \mathrm{~A} / \mathrm{FeGa})$ и $\mathrm{Fe}_{0,62} \mathrm{Co}_{0,19} \mathrm{Ga}_{0,19}(440 \mathrm{~A} / \mathrm{FeCoGa}) . H=58$ Э

Для ламинатных структур величина МЭКН пропорциональна отношению $d_{11} / \varepsilon_{11}$. Для пьезокерамики типа PZT относительная диэлектрическая проницаемость $\varepsilon_{11}$ слабо зависит от частоты в диапазоне до 10 кГц. Поэтому наблюдающиеся изменения МЭКН с частотой качественно могут быть объяснены частотными изменениями характеристик ферромагнитных слоев. Коэффициент магнитомеханической связи определяется как $k_{m}=\left(4 \pi \lambda^{\prime} \mu_{r}{ }^{\prime} / \mathrm{E}\right)^{1 / 2}$, где $\lambda^{\prime}-$ коэффициент магнитострикции в переменном магнитном поле, который в свою очередь пропорционален $q, \mu_{r}{ }^{\prime}$ - действительная часть относительной магнитной проницаемости; $E$ - модуль Юнга [13]. Наблюдаемое увеличение максимального значения $\alpha$ для всего исследованного диапазона частот для структур с пленкой $\mathrm{Fe}_{0,72} \mathrm{Ga}_{0,28}$ по-видимому связано с увеличением $k_{m}$ за счет $q$ из-за разных знаков $\lambda$ в аморфном сплаве и пленке.

Сильное уменьшение максимального значения $\alpha$ для структур $\mathrm{c}$ пленкой $\mathrm{Fe}_{0,62} \mathrm{Co}_{0,19} \mathrm{Ga}_{0,19}$, скорее всего, может быть связано с влиянием текстуры. Согласно данным работы [10] в структуре пленок системы Fe-Co-Ga, выращенных на стекле, наблюдается сосуществование объемно-центрированной кубической и объемно-центрированной тетрагональной фаз. При увеличении концентрации Со степень тетрагональных искажений уменьшается и имеет место формирование текстуры типа [110]. Формирование аналогичной текстуры также наблюдалось и в работе [14], в которой исследовали пленки системы Fe-Ga, полученные на кремнии с естественным слоем окисла. В пленках с текстурой типа [110] максимальное значение магнитострикции насыщения $\lambda_{S}$ может быть оценено из выражения [15]

$$
\Lambda_{S=} \alpha \lambda_{100}+(1-\alpha) \lambda_{111}
$$

где $\lambda_{100}$ и $\lambda_{111}-$ соответствующие значения для монокристалла; $\alpha=(2 / 5)-\ln C / 8$, а $C=$ $2 C_{44} /\left(C_{11}-C_{12}\right)$, где $C_{44}, C_{11}$ и $C_{12}$ - соответствующие упругие модули. По данным [16], значение $\alpha$ для пленок состава Fe-Co-Ga может составлять 0,095. В этом случае первое слагаемое в формуле будет существенно меньше второго и величина $\lambda_{S}$ будет в основном определяться $\lambda_{111}$ и сильно зависеть от текстуры пленки. Для выяснения влияния этих факторов будут проведены дополнительные структурные исследования пленок. 
Из данных рис. 2. и 3 можно сделать вывод, что для решения задач неразрушающего контроля предпочтительно использование композитных планарных структурах на основе ферромагнитных магнитострикционных аморфных лент сплава типа «Метглас» с пленкой $\mathrm{Fe}_{0,72} \mathrm{Ga}_{0,28}$ в условиях возбуждения переменным полем с частотой 110 Гц.

\section{4. Заключение}

Исследован магнитоэлектрический эффект (МЭ) в ламинатных структурах ферромагнетик/сегнетоэлектрик/ферромагнетик с композитными ферромагнитными магнитострикционными слоями, полученными импульсным лазерным осаждением тонких пленок $\mathrm{Fe}_{0,72} \mathrm{Ga}_{0,28}$ и $\mathrm{Fe}_{0,62} \mathrm{Co}_{0,19} \mathrm{Ga}_{0,19}$ с отрицательной магнитострикцией на аморфные ленты типа «Метглас» с положительной магнитострикцией. Нанесение тонких пленок $\mathrm{Fe}_{0,72} \mathrm{Ga}_{0,28}$ приводит к увеличению максимальной величины МЭКН в ламинатных структурах во всем исследованном диапазоне частот, что связано с увеличением коэффициента магнитомеханической связи структуры в целом за счет роста пьезомагнитного коэффициента в системе аморфная лента/тонкая пленка. Нанесение тонких пленок $\mathrm{Fe}_{0,62} \mathrm{Co}_{0,19} \mathrm{Ga}_{0,19}$ приводит к уменьшению максимальной величины МЭКН в ламинатных структурах. Это, по-видимому, обусловлено влиянием текстуры пленки на пьезомагнитный коэффициент. Для решения задач магнитного неразрушающего контроля наилучшую чувствительность обеспечивают ламинатные структуры с магнитострикционной пленкой $\mathrm{Fe}_{0,72} \mathrm{Ga}_{0,28}$ в условиях возбуждения переменным полем с частотой 110 Гц.

\section{Благодарность}

Работа выполнена в основном, на средства субсидии, выделенной на финансирование проектов Комплексной программы Уральского отделения РАН (проект № 15-9-2-30), и частично в рамках проекта РФФИ 15-02-01782.

\section{Литература}

1. Magnetic field sensors based in $\mathrm{Fe} / \mathrm{Cr}$ superlattices / L. Romashev, A. Rinkevich, A. Yuvchenko, A. Burkhanov // Sensors and Actuators A: Physical. - 2001. - Vol. 91, iss. 1-2. - P. 30-33. - DOI: 10.1016/S0924-4247(01)00482-4.

2. Hysteresis-free spin valves with a noncollinear configuration of magnetic anisotropy / L. I. Naumova, M. A. Milyaev, T. A. Chernyshova, V. V. Proglyado, I. Yu. Kamenskii, V. V. Ustinov // Physics of the Solid State. - Vol. 56, iss. 6. - P. 1125-1130. - DOI: $10.1134 /$ S 1063783414060274.

3. Lawes G., Srinivasan G. Introduction to magnetoelectric coupling and multiferroic films // J. Phys. D: Appl. Phys. - 2011. - Vol. 44. - P. 243001 (22). - DOI:10.1088/00223727/44/24/243001.

4. Petrov V. M., Srinivasan G. Enhancement of magnetoelectric coupling in functionally graded ferroelectric and ferromagnetic bilayers // Phys. Rev. B. - 2008. - Vol. 78. - P. 184421 (8). DOI: 10.1103/PhysRevB.78.184421.

5. Flexural deformation in a compositionally stepped ferrite and magnetoelectric effects in a composite with piezoelectrics / S. K. Mandal, G. Sreenivasulu, V. M. Petrov, G. Srinivasan // Appl. Phys. Lett. - 2010. - Vol. 96. - P. 192502 (3). - DOI: 10.1063/1.3428774.

6. Magnetic Alloy 2605SA1 (iron-based) // Tech. Bulletin. Ref: 2605SA106192009. - Metglas Inc., Conway, SC, 2009.

7. Out-of-plane anisotropy and low field induced magnetic domain reorientation in Al/Metglas-2605S2/Al trilayer sensors / E. C. Passamani, C. Larica, P. S. Moscon, P. Mendoza Zelis, F. H. Sanchez // J. Appl. Phys. - 2010. - Vol. 110. - P. 043906 (7). - DOI: 10.1063/1.3622339. 
8. ГМ440А. Магнитные свойства кольцевых магнитопроводов из аморфных и нанокристаллических сплавов в защитных контейнерах [Электронный pecypc]. - URL: http://www.gammamet.ru/ru/gm440a.htm.

9. Atulasimha Jayasimha, Flatau Alison B. Topical Review. A review of magnetostrictive iron-gallium alloys // Smart Materials and Structures. - 2011. - Vol. 20, no. 4. - P. 043001 (15). DOI:10.1088/0964-1726/20/4/043001.

10. Magnetostrictive and structural properties of FeCoGa films / S. U. Jen, T. L. Tsai, P. C. Kuo, W. L. Chi, W. C. Cheng // J. Appl. Phys. - 2010. - Vol. 107, iss. 1. - P. 013914 (4). DOI: $10.1063 / 1.3284962$.

11. OptoSystems. The leading manufacturer of lasers for medicine, science and technology in Russia. [Электронный ресурc]. - URL: http://www.optosystems.ru/eng/index.php.

12. Magnetoelectric interactions in layered composites of piezoelectric quartz and magnetostrictive alloys / G. Sreenivasulu, V. M. Petrov, L. Y. Fetisov, Y. K. Fetisov, G. Srinivasan // Physical Review B. - 2012. - Vol. 86. - P. 214405 (7). - DOI: 10.1103/PhysRevB.86.214405.

13. Van der Burgt C. M. Dynamical parameters in the magneto-strictive excitation of extensional and torsional vibrations in ferrites // Philips Res. Rep. - 1953. - Vol. 8. - P. 91-132.

14. Morley N. A., Javed A., Gibbs M. R. Effect of a forming field on the magnetic and structural properties of thin Fe-Ga films // J. Appl. Phys. - 2009. - Vol. 105. - P. 07A912 (4). - DOI: 10.1063/1.3059612.

15. Callen Herbert B., Goldberg N. Magnetostriction of Polycrystalline Aggregates // J. Appl. Phys. - 1965. - Vol. 36. - P. 976-977. - DOI: 10.1063/1.1714285.

16. Wuttig Manfred, Dai Liyang, Cullen J. Elasticity and magnetoelasticity of Fe-Ga solid solutions // App. Phys. Lett. - 2002. - Vol. 80. - P. 1135 (3). - DOI: 10.1063/1.1450045. 